\title{
Hyperbaric Oxygen Combined with Alginate Oligosaccharide Delay D-gal-induced Liver, Kidney and Spleen Senescence in Mice by Attenuating Oxidative Stress
}

\section{Gui-Mei Wang}

The Affiliated Hospital of Qingdao University

Xiao-yue Zheng

The Affiliated Hospital of Qingdao University

\section{Pei-Song Wang}

The Affiliated Hospital of Qingdao University

Jin-E Wan

The Affiliated Hospital of Qingdao University

Jian Gu

The Affiliated Hospital of Qingdao University

\section{Ming Chen}

The Affiliated Hospital of Qingdao University

\section{Ying Long}

Shenzhen People's Hospital

Yongjun Mao ( $\sim$ maoyongjun@qduhospital.cn )

The Affiliated Hospital of Qingdao University https://orcid.org/0000-0003-0359-2876

\section{Research Article}

Keywords: D-galactose, aging, mice, hyperbaric oxygen, alginate oligosaccharide

Posted Date: December 16th, 2021

DOI: https://doi.org/10.21203/rs.3.rs-1144553/v1

License: (c) (i) This work is licensed under a Creative Commons Attribution 4.0 International License. Read Full License 
Types of papers: Animal Cell Technology

\title{
Hyperbaric Oxygen Combined with Alginate Oligosaccharide Delay D-gal-induced Liver, Kidney and Spleen Senescence in Mice by Attenuating Oxidative Stress
}

Guimei Wang ${ }^{1 \#}$, Xiaoyue Zheng ${ }^{1 \#}$, Peisong Wang ${ }^{2}$, Jin'e Wan ${ }^{2}$, Jian $\mathrm{Gu}^{2}$, Ming Chen ${ }^{1}$, Ying Long ${ }^{3}$, Yongjun $\mathrm{Mao}^{1}$

${ }^{1}$ Department of Geriatric Medicine, The Affiliated Hospital of Qingdao University, Qingdao 266000, Shandong, China.

${ }^{2}$ Department of Hyperbaric Oxygen, The Affiliated Hospital of Qingdao University, Qingdao 266000, Shandong, China.

${ }^{3}$ Department of Hyperbaric Oxygen, Shenzhen People's Hospital, The Second Clinical Medical College of Jinan University, The First Affiliated Hospital of Southern University of Science and Technology, Shenzhen, 518202, Guangdong, China.

* Corresponding author. Ying Long. E-mail addresses: 13823730482@139.com.

** Corresponding author. Yongjun Mao. E-mail addresses: maoyongjun @qduhospital.cn.

\#Guimei Wang and Xiaoyue Zheng contributed equally to this work.

\begin{abstract}
Objectives Hyperbaric oxygen treatment (HBOT) is an adjuvant therapy progressively applied in the treatment of clinical diseases, and alginate oligosaccharide (AOS) is a natural polysaccharide with excellent antioxidant property. In this study, the effects of HBOT combined with AOS on the senescence of liver, kidney and spleen were investigated in a D-galactose (D-gal)-induced senescent mice model.
\end{abstract}


Results The findings indicated that HBOT combined with AOS could delay D-gal-induced aging of liver, kidney and spleen in mice. Specifically, HBOT combined with AOS improved the daily behavior and organ indices of mice. Biochemical and morphological analyses of all tissues indicated that HBOT combined with AOS markedly decreased the unusual elevation of ALT, AST and CREA induced by Dgal, alleviated pathological injury, and significantly enhanced the capacity of antioxidant defense system of mice, including increasing T-SOD, CAT and GSH -Px activities and decreasing MDA content. In addition, Western Blot results indicated that HBOT combined with AOS also inhibited the overexpression of aging-related proteins p53 and p16.

Conclusions To some extent, these findings suggest that HBOT combined with AOS has significant anti-senescence potential in D-gal-induced senescent mice, and the underlying factors may be correlated with the enhancement of antioxidant defense capacity.

Keywords D-galactose, aging, mice, hyperbaric oxygen, alginate oligosaccharide

\section{Introduction}

The World Health Organization predicts that the global population over the age of 60 will reach $22 \%$ of the total population in 2050. As the population and life expectancy increase, the incidence of agerelated heart(Song, Zhao, and Zou 2020), liver, kidney and other organ(Khosla et al. 2020) diseases increases. This may pose new challenges to the healthcare system(Newgard and Sharpless 2013). Therefore, there is an urgent need to discover drugs or clinical treatments to delay aging.

One of the causes of progressive aging in people is oxidative stress. Oxidative stress is the consequence of an unbalance between the formation of reactive oxygen species (ROS) and the antioxidative system of the body. (Palmer and Kitchin 2010; Ciacka et al. 2020). Many researchers have 
used D-galactose (D-gal)-induced senescent models to investigate senescence and organ damage. Continuous D-gal injections lead to changes resembling natural senescence (Huang et al. 2019), mainly due to the reduction of intracellular galactose to galactitol, which unable to further degraded. The accumulation of galactitol causes disturbances in cellular metabolism, especially in metabolism-related organs (liver and kidney) where the scavenging capacity exceeds that of antioxidant enzymes, generating excess ROS, eventually leading to aging(Chen et al. 2010; Liu et al. 2018).

Hyperbaric oxygen treatment (HBOT) is a clinical adjunct in which hyperoxia or pure oxygen is administered at a pressure higher than atmospheric pressure (usually at 2 to 3 atmospheres absolute)(Bennett et al. 2016). According to the recommendations of the Undersea and Hyperbaric Medical Society, HBOT is recommended as therapy for 14 diagnoses, including carbon monoxide poisoning, air/gas emboli, and other chronic wounds(Godman et al. 2010; Gill and Bell 2004). Studies have demonstrated that the beneficial effects of HBOT are primarily connected with the regulation of oxidative stress and aging-related gene expression(Chen, Li, et al. 2016; Chen, Chen, et al. 2016; Gamdzyk et al. 2016). HBOT has been used in studies of acute liver injury(Lv et al. 2016; Losada et al. 2014) and liver-related cancers(Peng et al. 2014). In addition, Ramalho et al. found that HBOT alleviated renal insufficiency, reduced blood urea nitrogen, serum creatinine, proteinuria, and partial excretion of sodium and potassium in a model of renal I / R injury, resulting in reduced histological damage(Ramalho et al. 2012). HBOT is also superior to other procedures in terms of function and viability in rats with autologous splenic implants(Paulo et al. 2010). More importantly, HBOT monotherapy or synergy with many conventional pharmacological therapies has been shown to improve treatment effects(Stepien, Ostrowski, and Matyja 2016; Teguh et al. 2016).

Alginate oligosaccharide (AOS) is a natural medicine derived from the degradation of alginate, 
has recently attracted much attention due to its role in slowing biological aging and damage in models(Xing et al. 2020). In previous studies by our group, AOS has been shown to delay cataracts in D-gal-induced senescent mice by modulating the antioxidant system(Feng, Yang, et al. 2021). In addition, Terakado et al. demonstrated that AOS treatment attenuated hypertension-induced renal damage, including hypertensive glomerulosclerosis and kidney artery damage, in Dahl salt-sensitive rats on a high-salt diet(Terakado et al. 2012). More importantly, one of our previous studies confirmed that AOS contributes to the attenuation of renal aging, which may be attributed to the activation of the Nrf2 pathway and the enhancement of endogenous antioxidant defense systems(Pan et al. 2021).

Based on previous studies of HBOT and AOS, we found that both could ameliorate free radical involvement in various tissues, thus there is reason to believe that HBOT combined with AOS may have therapeutic potential for age-related liver, kidney and spleen diseases. In the present research, we assessed the anti-aging effects of HBOT combined with AOS on the liver, kidney and spleen by using a D-gal-induced senescent mice model to provide a new therapeutic idea for the clinical therapy of aging-related liver, kidney and spleen damage.

\section{Materials and Methods}

\subsection{Drugs and Chemicals Reagents}

AOS was provided by Xi'an Kaipu Medical Laboratory Co. Ltd. (Xi'an, China). D-gal was supplied by Qingdao Yunshan Biotechnology Co. Ltd. (Qingdao, China). The alanine aminotransferase (ALT), aspartate transaminase (AST), and creatinine (CREA) levels detected by corresponding kits from Changchun Huili Biotechnology Co. Ltd. (Changchun, China). Oxidative stress-related kits for the measurements of total superoxide dismutase (T-SOD), catalase (CAT), and malondialdehyde (MDA) 
were purchased from the Nanjing Jiancheng Bioengineering Institute (Nanjing, China), glutathione peroxidase (GSH-Px) kit was supplied by Beyotime Biotechnology Co., Ltd. (Shanghai, China). BCA protein assay kit was purchased from Boster Biological Technology Co., Ltd. (Wuhan, China). The p53 antibody was supplied by Cell Signaling Technology (Beverly, MA, USA), the p16 antibody was supplied by Abcam Inc. (Cambridge, MA, USA), and the GAPDH antibody was supplied by Beijing Solarbio Technology Co. Ltd. (Beijing, China).

\subsection{Animals}

Male 3-month-old Kunming mice were supplied by Beijing SPF Biotechnology Co., LTD (Beijing, China). Mice were placed at $18-22^{\circ} \mathrm{C}$ under 12 -hour cyclic light conditions with relative humidity (4060\%). All animal experimental processes complied with the "Guide for the Care and Use of Laboratory Animals" of Qingdao University and were authorized by the Animal Experimentation Ethics Committee of Qingdao University. Throughout the experiment, the appearance and daily behavior of mice were observed daily, and weighed once a week. The mice were separated into five groups in a random manner (n=9): Control group, D-gal group, D-gal + HBOT group, D-gal + AOS group, D-gal + HBOT + AOS group. The treatments for each group are shown in Table 1. Blood and tissues were collected directly after the experiments. Serum was acquired after centrifugation at $3000 \mathrm{rpm}$ at $4^{\circ} \mathrm{C}$. The weights of liver, kidney and spleen were recorded and the organ index was computed based on the following equation: organ index $(\mathrm{mg} / \mathrm{g})=$ organ weight $(\mathrm{mg}) /$ body weight $(\mathrm{g})$.

\subsection{Hyperbaric oxygen treatment}

HBOT was performed in the hyperbaric oxygen department of the Affiliated Hospital of Qingdao 
University, and mice were positioned in a hyperbaric chamber. To reduce carbon dioxide accumulation, the mice were first ventilated with pure oxygen at a flow rate of $2.0 \mathrm{~L} / \mathrm{min}$, and then the pressure was raised to $0.20 \mathrm{MPa}$ at a rate of $100 \mathrm{Kpa} / \mathrm{min}$ for 60 minutes per treatment with pure oxygen. The decompression rate was consistent and lasted for 15 minutes.

Table 1. Treatment plans for experimental groups.

\begin{tabular}{cc}
\hline Group & Treatment \\
\hline Control & physiological saline $(5 \mathrm{ml} / \mathrm{kg} \cdot \mathrm{d})$, for 8 weeks \\
D-gal & D-gal $(200 \mathrm{mg} / \mathrm{kg} \cdot \mathrm{d})$, for 8 weeks \\
D-gal + HBOT & D-gal $(200 \mathrm{mg} / \mathrm{kg} \cdot \mathrm{d})$, for 8 weeks, HBOT was performed at week $7-8$ \\
D-gal + AOS & D-gal $(200 \mathrm{mg} / \mathrm{kg} \cdot \mathrm{d})+$ AOS $(150 \mathrm{mg} / \mathrm{kg} \cdot \mathrm{d})$, for 8 weeks \\
D-gal + HBOT + AOS & D-gal $(200 \mathrm{mg} / \mathrm{kg} \cdot \mathrm{d})+$ AOS $(150 \mathrm{mg} / \mathrm{kg} \cdot \mathrm{d})$, for 8 weeks, HBOT was \\
& performed at week $7-8$
\end{tabular}

\subsection{Measurement of liver and kidney function}

Melt the serum on ice, and serum levels of ALT, AST and CREA were measured by an automated biochemical analyzer (Shenzhen Rayto Life Science Co., Ltd., China).

\subsection{Histological Analysis.}

After execution, fresh mice liver, kidney and spleen tissues were rapidly obtained, fixed in $4 \%$ paraformaldehyde, then embedded in paraffin, sliced and stained with hematoxylin eosin (H\&E). Histopathological examination was examined under a light microscopy.

\subsection{Western blot analysis.}


The liver, kidney and spleen tissues were lysed in RIPA buffer and centrifuged at $12000 \mathrm{~g}$ for 15 minutes at $4^{\circ} \mathrm{C}$, then the protein supernatant was collected. The protein concentration was measured using the BCA Protein Assay Kit. Equal amounts of protein $(25 \mu \mathrm{g} / \mathrm{lane})$ were separated by $12.5 \%$ SDSpolyacrylamide gel electrophoresis and transferred onto PVDF membranes. The membranes were then incubated in $5 \%$ skim milk for 2 hours and incubated overnight at $4{ }^{\circ} \mathrm{C}$ with the following antibodies: p53, p16, GAPDH. GAPDH served as an internal control. Finally, the membranes were incubated with secondary antibodies for 2 hours at room temperature, and the bands were visualized by gel imaging system using chemiluminescence detection reagents and measured by Image $\mathbf{J}$ software.

\subsection{Antioxidant index of liver, kidney and spleen}

The liver, kidney and spleen tissues of the mice were quickly removed, homogenized in PBS at a ratio of 1:9 and centrifuged. The protein concentrations were determined by using the BCA protein assay kit, and the activities of T-SOD, CAT and GSH-Px and the level of MDA in the tissues were measured according to the manufacturer's instructions.

\subsection{Statistical Analysis.}

All data were obtained from the results of three independent experiments. Statistical analysis was performed using SPSS 21.0 software (SPSS, Chicago, USA) and data are expressed as mean \pm SEM. One-way Analysis of Variance (ANOVA) was used for multiple group comparisons and LeastSignificant Difference (LSD) method was used for comparison between groups. $P$-values $<0.05$ were considered to be statistically significant. 


\section{Results}

\subsection{Daily behavior and Body weight.}

The daily behavior and appearance of the mice were observed daily throughout the experimental period. The mice in the control group had good appetite, smooth hair and agile movement. The mice in the D-gal injection group were gradually depressed, unresponsive, with reduced appetite and dull hair, showing the characteristics of aging mice, which were significantly alleviated with the AOS and HBOT treatment groups. In addition, the results of body weight are shown in Fig.1, the mice in the control group started to gain weight slowly and then gained weight steadily, while the mice administered with D-gal gained weight more slowly compared to the control group. Starting from week 4, the mice administered with AOS gradually resumed body weight gain, and the mice in the HBOT group also gradually resumed body weight gain at 7-8 weeks after receiving the treatment.

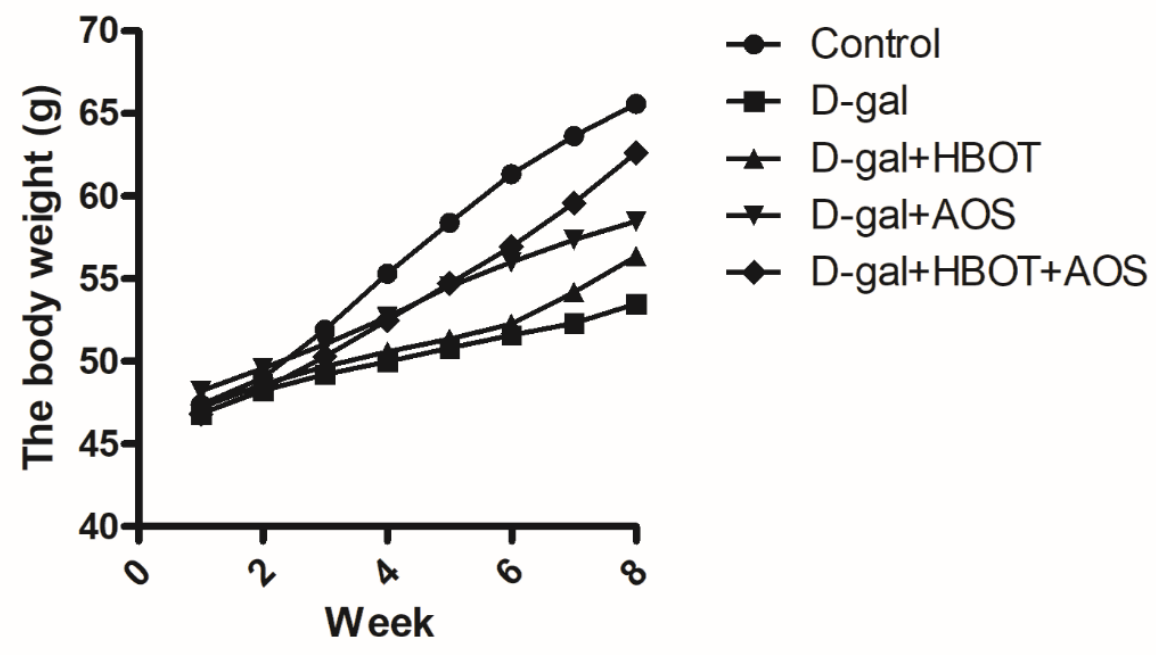

Fig.1 Weight of mice in each group during the experiment.

\subsection{Effect of HBOT and AOS on organ indices.}

We further analyzed the organ indices of the mice. The results are shown in Table 2. The organ 
indices of liver, kidney and spleen were markedly lower in the D-gal group than the control group $(p<$ 0.01), which may be related to organ atrophy caused by D-gal injection, while HBOT and AOS could improve the organ indices of liver, kidney and spleen in mice $(p<0.01)$, and the combination of the two was even better $(p<0.01)$, with organ indices closest to those of the control group. These findings suggest that HBOT and AOS could improve the decrease of organ index in D-gal-induced senescent mice, and the combination of the two has a more pronounced effect on delaying organ aging.

Table 2. Effects of HBOT and AOS on the organ indices in mice of each group.

\begin{tabular}{cccc}
\hline Groups & Liver index & Kidney index & Speen index \\
& $(\mathbf{m g} / \mathbf{g})$ & $(\mathbf{m g} / \mathbf{g})$ & $(\mathbf{m g} / \mathbf{g})$ \\
\hline Control & $50.12 \pm 0.46$ & $20.33 \pm 0.29$ & $7.88 \pm 0.06$ \\
D-gal & $37.27 \pm 0.46^{\mathrm{a}}$ & $13.19 \pm 0.20^{\mathrm{a}}$ & $5.54 \pm 0.06^{\mathrm{a}}$ \\
D-gal + HBOT & $42.31 \pm 0.37^{\mathrm{bc}}$ & $15.48 \pm 0.26^{\mathrm{bc}}$ & $6.22 \pm 0.07^{\mathrm{bc}}$ \\
D-gal + AOS & $43.73 \pm 0.64^{\mathrm{bc}}$ & $15.86 \pm 0.24^{\mathrm{bc}}$ & $6.45 \pm 0.09^{\mathrm{bc}}$ \\
D-gal + HBOT + AOS & $46.33 \pm 0.54^{\mathrm{b}}$ & $18.43 \pm 0.30^{\mathrm{b}}$ & $7.25 \pm 0.08^{\mathrm{b}}$ \\
\hline
\end{tabular}

Note: ${ }^{\mathrm{a}} p<0.01$ vs. Control group; ${ }^{\mathrm{b}} p<0.01$ vs. D-gal group; ${ }^{\mathrm{c}} p<0.01$ vs. D-gal + HBOT + AOS group.

\subsection{Effect of HBOT and AOS on liver and kidney function}

We assessed the extent of liver and kidney damage in mice by measuring the serum levels of ALT, AST and CREA. As shown in Table 3, compared with the control group, the levels of ALT, AST and CREA in the serum of the D-gal group were remarkably enhanced $(p<0.01)$, and the levels of ALT, AST and CREA in the serum of mice given AOS and HBOT treatment groups were decreased to some extent compared with the D-gal group $(p<0.01)$, while the levels of ALT, AST and CREA in the serum of mice treated with the combination of AOS and HOBT were greatly declined $(p<0.01)$. It showed 
that the combination of HBOT and AOS could significantly promote the recovery of liver and kidney function in D-gal-induced senescent mice.

Table 3. Effects of HBOT and AOS on liver and kidney functions in mice of each group.

\begin{tabular}{cccc}
\hline Groups & \multicolumn{2}{c}{ Liver Function } & Kidney Function \\
\cline { 2 - 4 } & ALT $(\mathbf{U} / \mathbf{L})$ & AST $(\mathbf{U} / \mathbf{L})$ & CREA $(\boldsymbol{\mu m o l} / \mathbf{L})$ \\
\hline Control & $28.26 \pm 3.32$ & $108.23 \pm 5.25$ & $20.72 \pm 1.50$ \\
D-gal & $70.02 \pm 6.42^{\mathrm{a}}$ & $175.52 \pm 7.83^{\mathrm{a}}$ & $36.93 \pm 2.84^{\mathrm{a}}$ \\
D-gal + HBOT & $50.79 \pm 5.22^{\mathrm{bc}}$ & $149.66 \pm 6.61^{\mathrm{bc}}$ & $29.70 \pm 1.64^{\mathrm{bc}}$ \\
D-gal + AOS & $47.51 \pm 3.06^{\mathrm{bc}}$ & $137.03 \pm 6.70^{\mathrm{bc}}$ & $27.91 \pm 0.78^{\mathrm{bc}}$ \\
D-gal + HBOT + AOS & $37.88 \pm 1.90^{\mathrm{b}}$ & $122.78 \pm 3.79^{\mathrm{b}}$ & $25.23 \pm 0.90^{\mathrm{b}}$ \\
\hline
\end{tabular}

Note: ${ }^{\mathrm{a}} p<0.01$ vs. Control group; ${ }^{\mathrm{b}} p<0.01$ vs. D-gal group; ${ }^{\mathrm{c}} p<0.01$ vs. D-gal + HBOT + AOS group.

\subsection{Protective effects of HBOT and AOS on D-gal-induced pathological histological damage in}

\section{liver, kidney and spleen}

H\&E staining could directly reflect the extent of injury to the liver, kidney and spleen. As shown in Fig.2, the liver histological structure of the mice in control group are clearly with regular morphology, uniform size hepatocytes and radially-ranged hepatic cords surrounding the central vein.

The mice injected with D-gal for 8 weeks exhibited obvious damage to liver structure, including an increase in hepatic binucleated cells, looser cell arrangement and larger cell gaps, along with a certain degree of ballooning degeneration and inflammatory cell infiltration. In contrast, both HBOT and AOS treatment groups showed superior hepatoprotective effects, and more importantly, liver damage was significantly improved in the combined treatment group of both. 


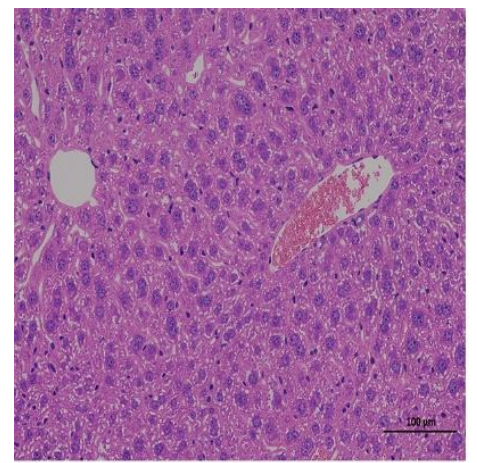

Control

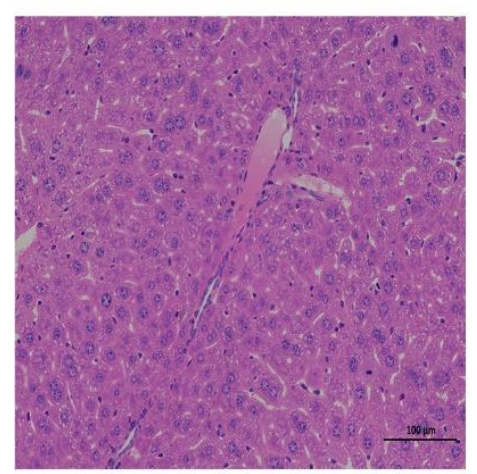

D-gal + AOS

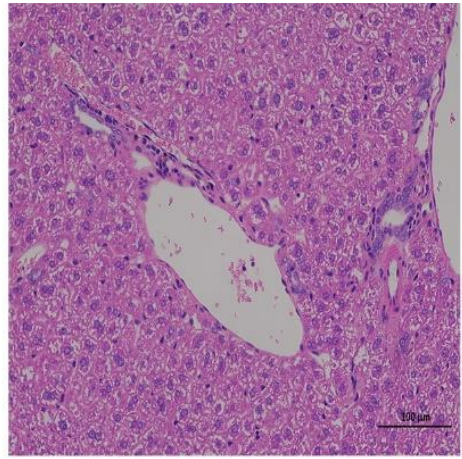

D-gal

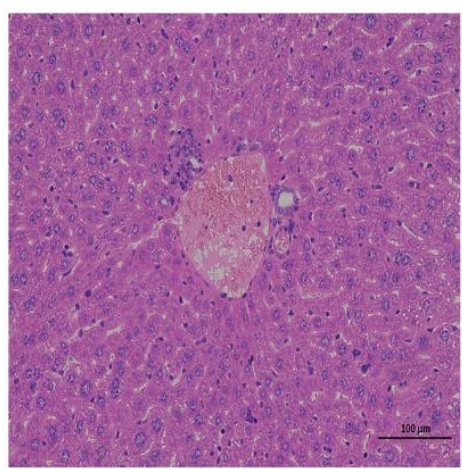

$\mathrm{D}-\mathrm{gal}+\mathrm{HBOT}+\mathrm{AOS}$

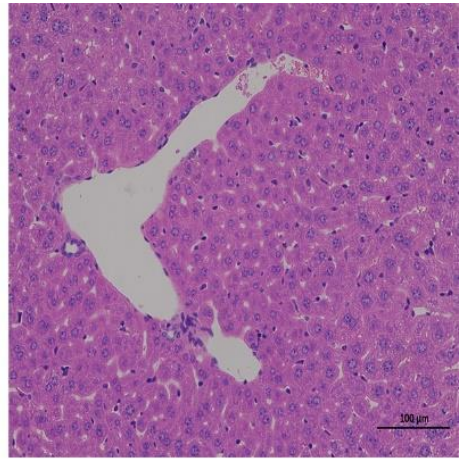

D-gal + HBOT

Fig.2 Pathological observation of H\&E in the liver of each group of mice. $(200 \times)$

Similarly, as shown in Fig.3, the kidney structure of the control mice was normal, while the mice in the D-gal group exhibited significant kidney damage with marked glomerular atrophy, balloon widened cavity, and reduction of epithelial cells in renal tubules. Compared with the D-gal group, HBOT and AOS treatment alleviated the above kidney lesions induced by D-gal, thus protecting the kidneys, and notably, the combination of the two alleviated them more significantly. 


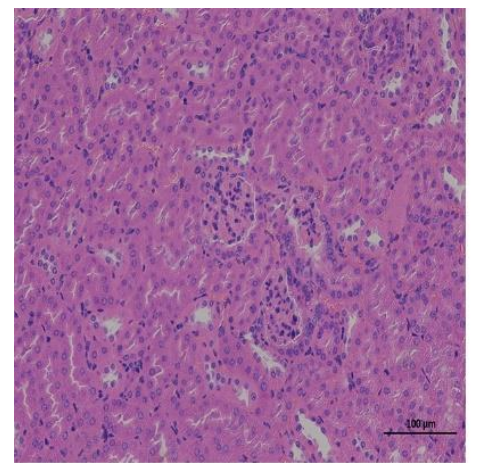

Control

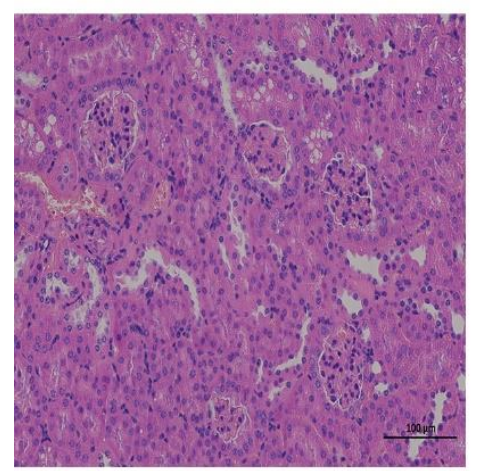

D-gal + AOS

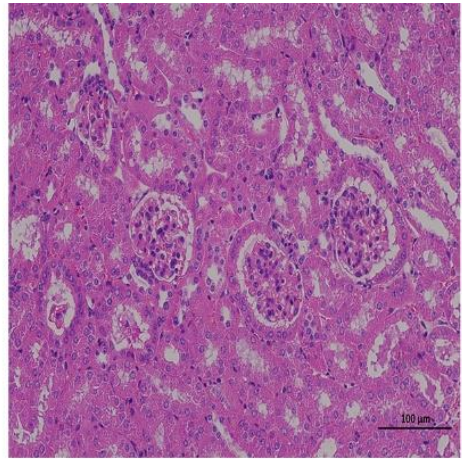

D-gal

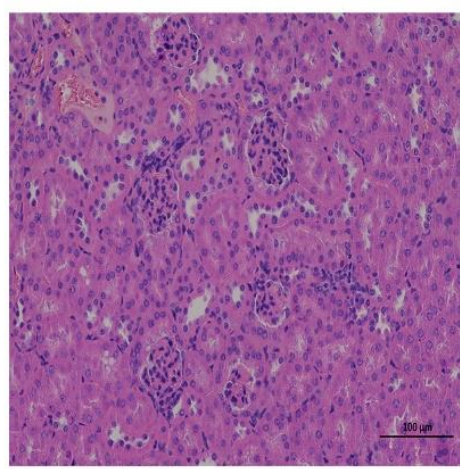

D-gal + HBOT + AOS

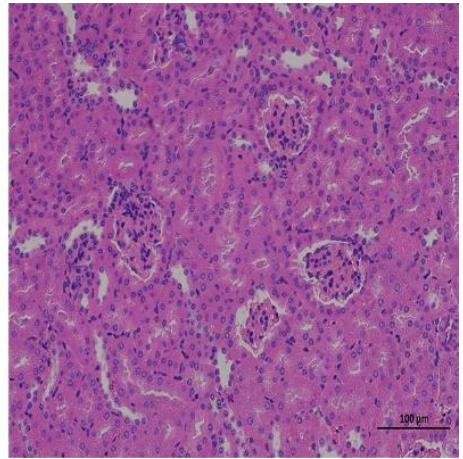

D-gal + HBOT

Fig.3 Pathological observation of H\&E in the kidney of each group of mice. $(200 \times)$

Fig.4 shows the histological photographs of the spleen. The control mice had normal spleen trabeculae structure, and the boundary between the red and white pulp was clear, while the spleen histomorphology of mice induced by D-gal was irregular, with blurred border between red and white pulp and reduced cell density. After treatment with HBOT and AOS, the spleen histomorphology of mice was significantly improved, with the combination of the two having the best effect. In conclusion, it can be shown that HBOT and AOS could improve the morphological damage of liver, kidney and spleen in D-gal-induced senescent mice, and the effect is better after combining the two treatment modalities. 


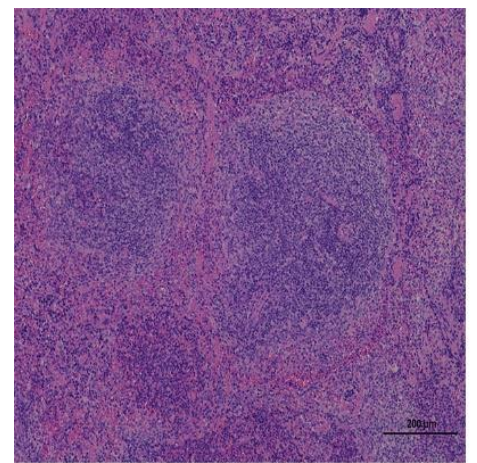

Control

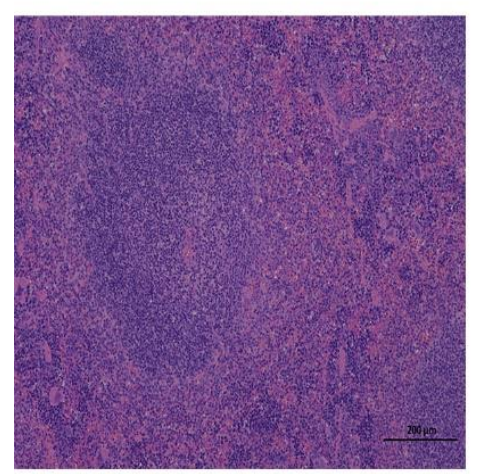

D-gal + AOS

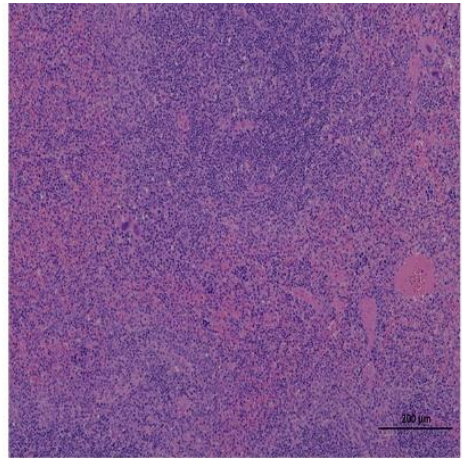

D-gal

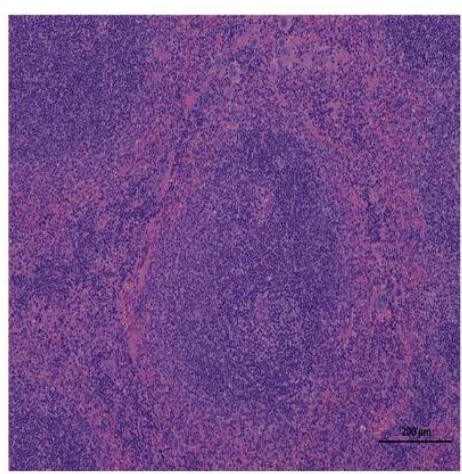

$\mathrm{D}-\mathrm{gal}+\mathrm{HBOT}+\mathrm{AOS}$

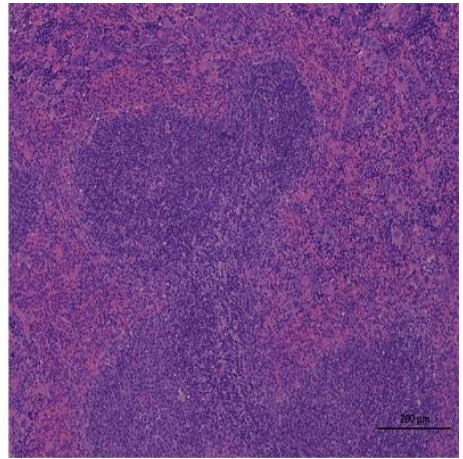

D-gal + HBOT

Fig.4 Pathological observation of H\&E in the spleen of each group of mice. $(100 \times)$

\subsection{Effects of HBOT and AOS on aging-related markers in the liver, kidney and spleen}

We examined the expression of common senescence-related markers p53 and p16 in liver, kidney and spleen tissues by Western blot, and the results are shown in Fig.5 Compared with the control group, the expression of p53 and p16 was increased in liver, kidney and spleen of D-gal-treated mice $(p<$ 0.01), suggesting that D-gal-induced senescence may be induced by stimulating the key factors p53 and p16. The Western blot results also indicated that the protein expression levels of p53 and p16 were regulated down in the HBOT and AOS treatment groups $(p<0.01-0.05)$, with the most significant down-regulation in the combined treatment group $(p<0.01-0.05)$, which further confirmed the antiaging effect of HBOT combined with AOS in liver, kidney and spleen. 
$\mathrm{a}$

(Liver)
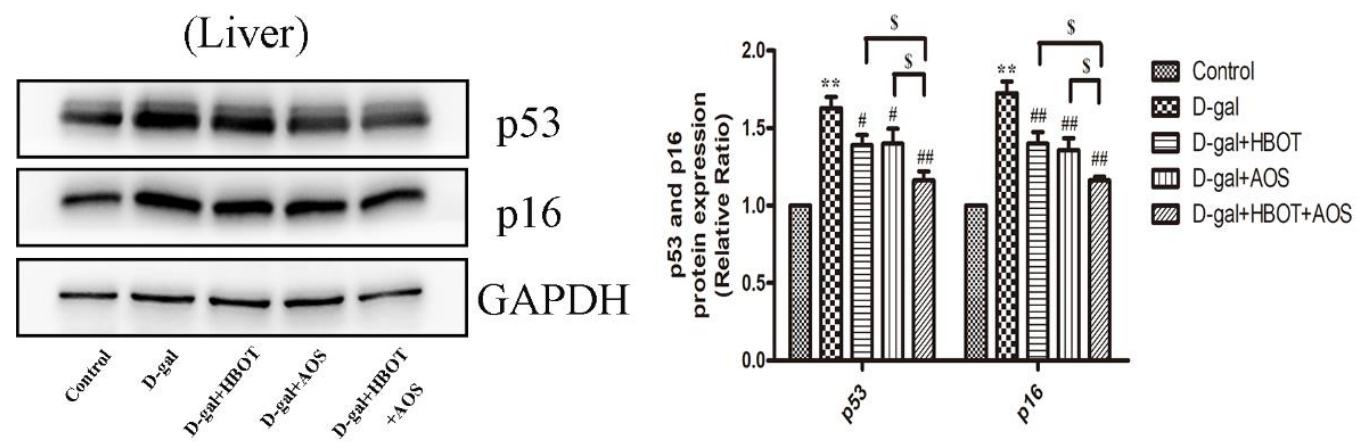

$\mathrm{b}$
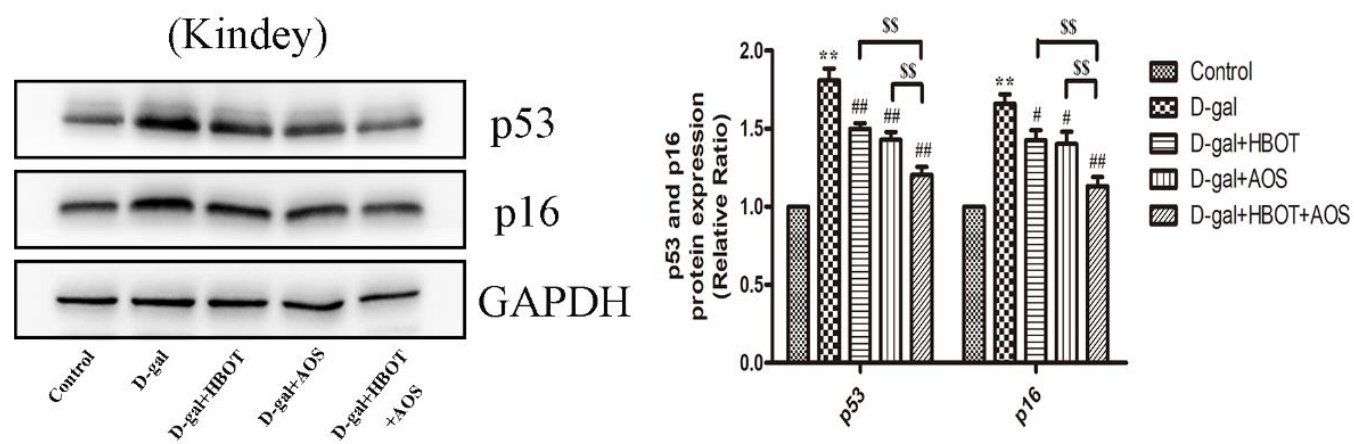

$\mathrm{C}$

(Spleen)
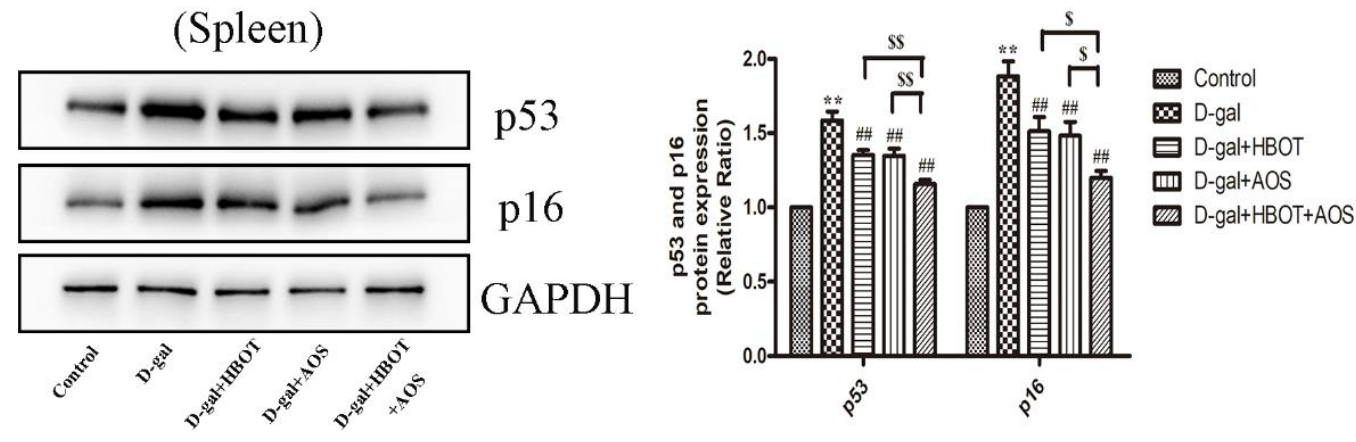

Fig.5 HBOT and AOS delay D-gal-induced liver, kidney and spleen senescence by inhibiting the key activation factors $\mathrm{p} 53$ and $\mathrm{p} 16$. Western blot was used to determine $\mathrm{p} 53$ and p16 protein expression (ac). GAPDH served as loading control. $* * p<0.01$ vs. Control, \#p $<0.05$ vs. D-gal, \#\#p $<0.01$ vs. Dgal, $\$ p<0.05$ vs. D-gal + HBOT + AOS, $\$ \$ p<0.01$ vs. D-gal + HBOT + AOS.

\subsection{Effect of HBOT and AOS on antioxidant indexes in liver, kidney and spleen}

The activities of T-SOD, CAT, GSH-Px and the content of MDA in the liver, kidney and spleen of mice were comprehensively analyzed by oxidative stress kit. The results are shown in Fig.6 Prolonged D-gal injection resulted in a distinct decline in the activities of T-SOD, CAT and GSH-Px, and a 
significant increase MDA level in the liver, kidney and spleen of mice, which were obvious changes from the control group $(p<0.01)$. This situation was improved in mice treated with HBOT and AOS $(p$ $<0.01$ ), and the activities of T-SOD, CAT and GSH-Px were markedly increased and MDA level were significantly decreased after the combination of both treatments $(p<0.01)$. These findings indicated that HBOT combined with AOS could significantly increase the expression of antioxidant enzymes in liver, kidney and spleen of mice and ameliorate the oxidative stress injury induced by D-gal in these tissues.
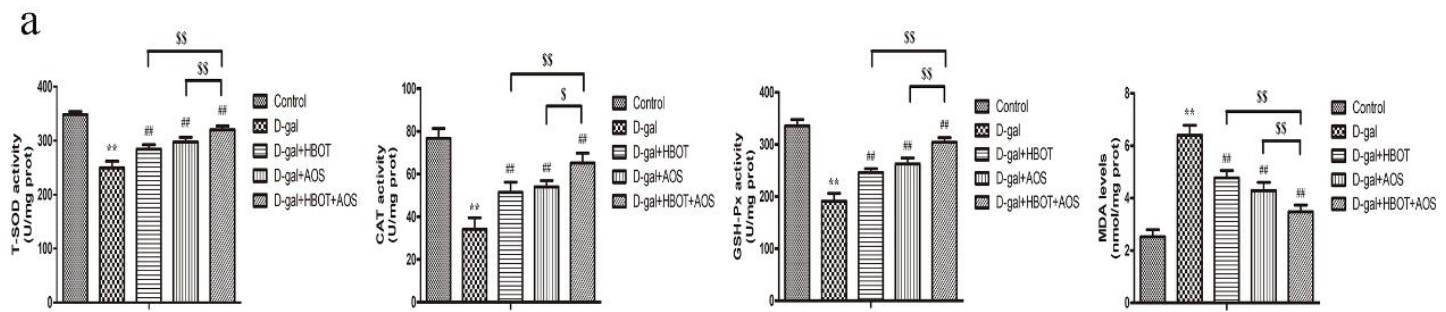

b
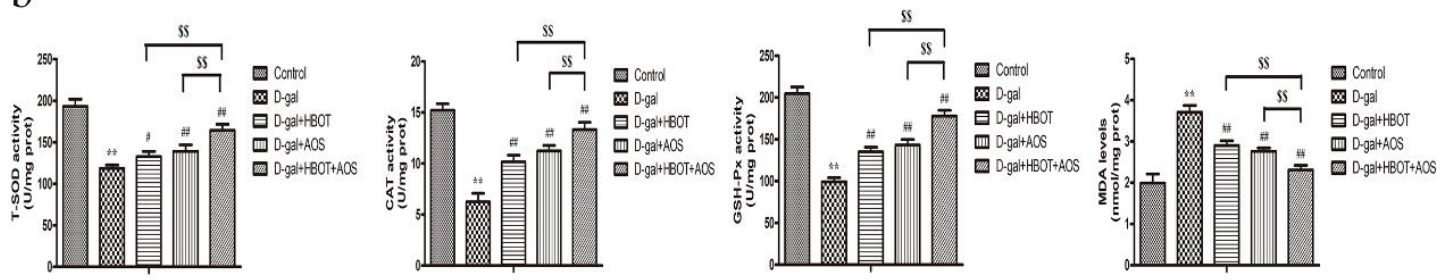

$\mathrm{c}$
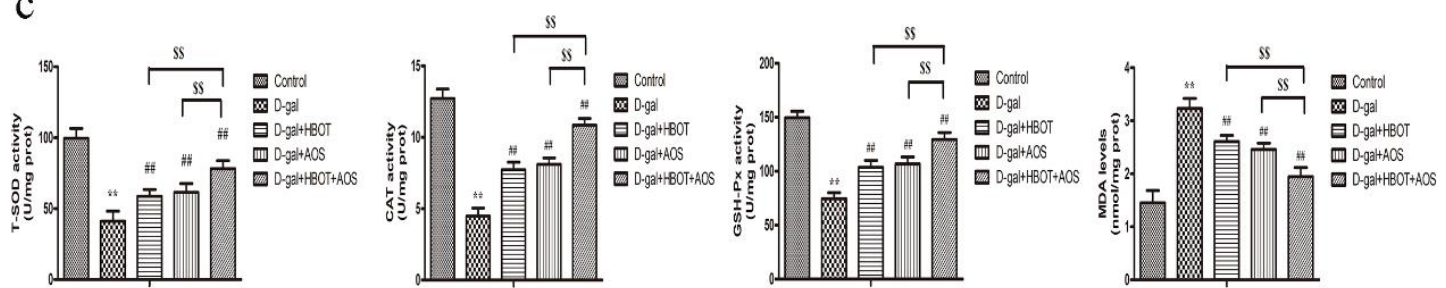

Fig.6 Effect of HBOT and AOS on the activities of T-SOD, CAT and GSH-Px and MDA levels in the liver (a), kidney (b) and spleen (c) of D-gal-induced mice. ${ }^{*} p<0.01$ vs. Control, $\# p<0.05$ vs. D-gal, $\# \# p<0.01$ vs. D-gal, $\$ p<0.05$ vs. D-gal + HBOT + AOS, $\$ \$ p<0.01$ vs. D-gal + HBOT + AOS.

\section{Discussion}


Aging is a multifactorial and complex phenomenon(Galkin et al. 2019). Previous studies by our group have indicated that AOS could delay D-gal-induced senescent in the heart(Feng, Liu, et al. 2021) and kidney(Pan et al. 2021) of mice, and other previous studies have also indicated that pretreatment with HBOT could increase the lifespan of Caenorhabditis elegans(Cypser and Johnson 2002). Nevertheless, there are no studies on HBOT combined with AOS to delay D-gal-induced aging in liver, kidney and spleen of mice. D-gal-induced senescent model is a common model of senescence, which is based on the principle of inducing metabolic disorders in animals(Gong et al. 2016). Almost all organs are susceptible to oxidative injury caused by D-gal, and the hepatic and renal are the main organs involved in D-gal metabolism, thus the hepatic and renal organs are more susceptible to D-gal-induced injury(Yu et al. 2015; Li et al. 2019; Li et al. 2016).

In the present research, a remarkable change in body weight was noted between the control group and the D-gal group, while it could be observed that the D-gal mice were sluggish and had a lusterless hair, which is consistent with the findings of Tian et al.(Tian et al. 2021). Several studies have shown that aging is associated with changes in organ indices(James et al. 2015), and changes in organ indices are one of the most important indicators of organ function(Sun et al. 2013). The results of the present research revealed that the organ indices of liver, kidney and spleen were obviously declined in the Dgal group, suggesting that D-gal could cause decline and atrophy of organ functions in mice. Whereas, both HBOT and AOS could effectively improve all organ indexes, and the combined effect was better, which indicated that HBOT combined with AOS could play a role in attenuating the aging effect of liver, kidney and spleen caused by D-gal.

Prior researches have demonstrated that long-term D-gal injections lead to liver and kidney damage and functional abnormalities(Park et al. 2015). The hepatic and renal organs are crucial organs 
for detoxification and excretion, and oxidative stress progressively impair the hepatic and renal organs with age, leading to the cumulation of various toxins substances(Hadzi-Petrushev et al. 2015; Indo et al. 2015). In particular, ALT and AST are the most common markers of changes in liver function, while CREA is a sensitive indicator of the degree of kidney damage(Zhang et al. 2019; Mo et al. 2017). Therefore, serological tests are essential to analyze liver and kidney function. After 8 weeks of D-gal injection, we could find that serum ALT, AST and CREA levels were significantly elevated, but HBOT and AOS treatment reduced the exceptional rises in serum ALT, AST and CREA, and the combination of the two reduced them more significantly. As studied by $\mathrm{C}$ et al, a significant reduction in some markers of renal damage could be found in the HBOT group(Verma et al. 2015). Therefore, HBOT combined with AOS has a protective effect against hepatic and renal injury in the mice model of D-gal aging.

Pathological observation is a method of identifying pathological changes in the organism by observing changes in the morphology of tissues or cells(Di Meo et al. 2016). H\&E staining of the liver, kidney and spleen can indicate a number of pathological features that occur in vivo due to aging(Qian et al. 2018). The results suggested that the organ morphology of the control mice was normal, while the liver, kidney and spleen structures of mice in the D-gal group were obviously damaged, showing a series of changes such as increased hepatic binucleated cells, significantly atrophied glomeruli, widened balloon cavity, and blurred border between red and white pulp in the spleen. The application of HBOT and AOS ameliorated D-gal-induced liver, kidney and spleen injury to some degree, and the morphological organization of mice treated with the combination of HBOT and AOS was closest to the control group. This is analogous to the conclusion of the study by Li et al.(Li et al. 2020), suggesting that this could be related to the antioxidant effects of HBOT and AOS. 
It is well known that p53, a key player in the senescence process, controls cell growth and apoptosis(He et al. 2017; Kang et al. 2015), while p16, a regulator of cellular aging, is associated with multiple age-related organ dysfunctions(Yzydorczyk et al. 2019; Ferenbach and Bonventre 2016). Cellular senescence could be induced through multiple cellular signaling pathways, where activation of p53 may contribute to aging through a variety of downstream targets, and upregulation of p53 causes G1 and G2 cell cycles arrest(Shchors et al. 2013; Rufini et al. 2013). In the present report, we observed a remarkable increase in p53 and p16 protein expression in the liver, kidney and spleen of the D-gal group, while HBOT and AOS treatment reduced p53 and p16 protein expression, and the combination of both reduced it more significantly. It suggests that HBOT combined with AOS could delay D-galinduced hepatic, renal and splenic senescence in mice.

Several evidences have shown that overproduction of ROS causes oxidative damage in tissues and impairs cellular metabolism, especially in organs with rapid metabolic processes(Zhuang et al. 2017; Shen et al. 2018; Zhen et al. 2016). The degree of oxidative injury could be assessed by the activity of antioxidant enzymes and markers of oxidative stress(Wang et al. 2020). Previous studies by our group have demonstrated the antioxidant and radical scavenging activities of AOS(Zhao et al. 2020), and the results of Ozden et al. have shown that HBOT could reduce MDA content and improve antioxidant activity, including the activities of T-SOD and GSH-Px(Ozden et al. 2004). SOD, one of the active substances widespread in organisms, is the primary antioxidant enzyme of the organism, which could facilitate the rapid conversion of superoxide to $\mathrm{H}_{2} \mathrm{O}_{2}$ (Kowald et al. 2012), while CAT and GSH-Px could further degrade $\mathrm{H}_{2} \mathrm{O}_{2}$ molecules to $\mathrm{H}_{2} \mathrm{O}$ (Wang et al. 2012). Notably, CAT levels are high in the liver(Mamalis et al. 2013). They are considered as the first line of defense against ROS(Tsai and Yin 2012). MDA is a toxic by-product of toxic lipid peroxidation induced by oxidative damage, which is 
considered not only as a marker of oxidative damage and ROS accumulation, but also as an indicator of senescence(Conti et al. 2016), and the accumulation of MDA may cause structural and functional degradation of cellular proteins and antioxidant enzymes(Yang et al. 2019). In agreement with these findings, we observed that 8 consecutive weeks of D-gal injection resulted in a marked rise in MDA levels and a noticeable reduction in T-SOD, CAT and GSH-Px activities. Interestingly, HBOT and AOS treated mice differentially enhanced the antioxidant enzyme activities of organs and reduced lipid peroxidation, as evidenced by enhanced T-SOD, CAT and GSH-Px activities and reduced MDA levels, with more pronounced effects in the HBOT combined with AOS treated group. This further confirmed that the antioxidant capacity of HBOT combined with AOS attenuated the oxidative stress in liver, kidney and spleen.

In summary, the present findings suggest that HBOT combined with AOS could delay the aging of liver, kidney and spleen in mice by modulating the endogenous antioxidant defense system. This is the first report on the beneficial effects of HBOT combined with AOS on D-gal-induced senescence and damage in liver, kidney and spleen. The present study provides a new therapy strategy for delaying age-related liver, kidney and spleen diseases with a combination of agents and clinical adjuvant therapy.

Acknowledgements This work was supported by National Natural Science Foundation of China (No 31571829)

\section{Declarations}

Conflict of interest The authors declare that they have no conflict of interest. 
Ethical approval The research protocols were approved by the Committee on the Ethics of Animal Experiments of Qingdao University (QYFY WZLL 26529). This article does not contain any studies with humans performed by any of the authors.

\section{References}

Bennett, M. H., J. Feldmeier, N. B. Hampson, R. Smee, and C. Milross. 2016. 'Hyperbaric oxygen therapy for late radiation tissue injury', Cochrane Database Syst Rev, 4: CD005005.http://dx.doi.org/10.1002/14651858.CD005005.pub4

Chen, B., Y. Zhong, W. Peng, Y. Sun, and W. J. Kong. 2010. 'Age-related changes in the central auditory system: comparison of D-galactose-induced aging rats and naturally aging rats', Brain Res, 1344: 43-53.http://dx.doi.org/10.1016/j.brainres.2010.04.082

Chen, C., W. Chen, Z. Nong, Y. Ma, S. Qiu, and G. Wu. 2016. 'Cardioprotective Effects of Combined Therapy with Hyperbaric Oxygen and Diltiazem Pretreatment on Myocardial IschemiaReperfusion Injury in Rats', Cell Physiol Biochem, 38: 201529.http://dx.doi.org/10.1159/000445561

Chen, X., Y. Li, W. Chen, Z. Nong, J. Huang, and C. Chen. 2016. 'Protective Effect of Hyperbaric Oxygen on Cognitive Impairment Induced by D-Galactose in Mice', Neurochem Res, 41: 3032-41.http://dx.doi.org/10.1007/s11064-016-2022-x

Ciacka, K., M. Tyminski, A. Gniazdowska, and U. Krasuska. 2020. 'Carbonylation of proteins-an element of plant ageing', Planta, 252: 12.http://dx.doi.org/10.1007/s00425-020-03414-1

Conti, V., V. Izzo, G. Corbi, G. Russomanno, V. Manzo, F. De Lise, A. Di Donato, and A. Filippelli. 
2016. 'Antioxidant Supplementation in the Treatment of Aging-Associated Diseases', Front Pharmacol, 7: 24.http://dx.doi.org/10.3389/fphar.2016.00024

Cypser, J. R., and T. E. Johnson. 2002. 'Multiple stressors in Caenorhabditis elegans induce stress hormesis and extended longevity', J Gerontol A Biol Sci Med Sci, 57: B10914.http://dx.doi.org/10.1093/gerona/57.3.b109

Di Meo, S., T. T. Reed, P. Venditti, and V. M. Victor. 2016. 'Role of ROS and RNS Sources in Physiological and Pathological Conditions', Oxid Med Cell Longev, 2016: 1245049.http://dx.doi.org/10.1155/2016/1245049

Feng, W., J. Liu, S. Wang, Y. Hu, H. Pan, T. Hu, H. Guan, D. Zhang, and Y. Mao. 2021. 'Alginate oligosaccharide alleviates D-galactose-induced cardiac ageing via regulating myocardial mitochondria function and integrity in mice', $J$ Cell Mol Med, 25: 715768.http://dx.doi.org/10.1111/jcmm.16746

Feng, W., X. Yang, M. Feng, H. Pan, J. Liu, Y. Hu, S. Wang, D. Zhang, F. Ma, and Y. Mao. 2021. 'Alginate Oligosaccharide Prevents against D-galactose-mediated Cataract in C57BL/6J Mice via Regulating Oxidative Stress and Antioxidant System', Curr Eye Res, 46: 80210.http://dx.doi.org/10.1080/02713683.2020.1842456

Ferenbach, D. A., and J. V. Bonventre. 2016. 'Acute kidney injury and chronic kidney disease: From the laboratory to the clinic', Nephrol Ther, 12 Suppl 1: S418.http://dx.doi.org/10.1016/j.nephro.2016.02.005

Galkin, F., B. Zhang, S. E. Dmitriev, and V. N. Gladyshev. 2019. 'Reversibility of irreversible aging', Ageing Res Rev, 49: 104-14.http://dx.doi.org/10.1016/j.arr.2018.11.008

Gamdzyk, M., M. Malek, E. Bratek, A. Koks, K. Kaminski, A. Ziembowicz, and E. Salinska. 2016. 
'Hyperbaric oxygen and hyperbaric air preconditioning induces ischemic tolerance to transient forebrain ischemia in the gerbil', Brain Res, 1648: 25765.http://dx.doi.org/10.1016/j.brainres.2016.07.025

Gill, A. L., and C. N. Bell. 2004. 'Hyperbaric oxygen: its uses, mechanisms of action and outcomes', QJM, 97: 385-95.http://dx.doi.org/10.1093/qjmed/hch074

Godman, C. A., K. P. Chheda, L. E. Hightower, G. Perdrizet, D. G. Shin, and C. Giardina. 2010. 'Hyperbaric oxygen induces a cytoprotective and angiogenic response in human microvascular endothelial cells', Cell Stress Chaperones, 15: 431-42.http://dx.doi.org/10.1007/s12192-009$\underline{0159-0}$

Gong, Y. S., J. Guo, K. Hu, Y. Q. Gao, B. J. Xie, Z. D. Sun, E. N. Yang, and F. L. Hou. 2016. 'Ameliorative effect of lotus seedpod proanthocyanidins on cognitive impairment and brain aging induced by D-galactose', Exp Gerontol, 74: 218.http://dx.doi.org/10.1016/j.exger.2015.11.020

Hadzi-Petrushev, N., V. Stojkovski, D. Mitrov, and M. Mladenov. 2015. 'D-galactose induced changes in enzymatic antioxidant status in rats of different ages', Physiological Research, 64: 6170.http://dx.doi.org/10.33549/physiolres.932786

He, L., Y. Chen, J. Feng, W. Sun, S. Li, M. Ou, and L. Tang. 2017. 'Cellular senescence regulated by SWI/SNF complex subunits through p53/p21 and p16/pRB pathway', Int J Biochem Cell Biol, 90: 29-37.http://dx.doi.org/10.1016/j.biocel.2017.07.007

Huang, J. L., C. Yu, M. Su, S. M. Yang, F. Zhang, Y. Y. Chen, J. Y. Liu, Y. F. Jiang, Z. G. Zhong, and D. P. Wu. 2019. 'Probucol, a "non-statin" cholesterol-lowering drug, ameliorates D-galactose induced cognitive deficits by alleviating oxidative stress via Keap1/Nrf2 signaling pathway in 
mice', Aging (Albany NY), 11: 8542-55.http://dx.doi.org/10.18632/aging.102337

Indo, H. P., H. C. Yen, I. Nakanishi, K. Matsumoto, M. Tamura, Y. Nagano, H. Matsui, O. Gusev, R. Cornette, T. Okuda, Y. Minamiyama, H. Ichikawa, S. Suenaga, M. Oki, T. Sato, T. Ozawa, D. K. Clair, and H. J. Majima. 2015. 'A mitochondrial superoxide theory for oxidative stress diseases and aging', J Clin Biochem Nutr, 56: 1-7.http://dx.doi.org/10.3164/jcbn.14-42

James, E. L., R. D. Michalek, G. N. Pitiyage, A. M. de Castro, K. S. Vignola, J. Jones, R. P. Mohney, E. D. Karoly, S. S. Prime, and E. K. Parkinson. 2015. 'Senescent human fibroblasts show increased glycolysis and redox homeostasis with extracellular metabolomes that overlap with those of irreparable DNA damage, aging, and disease', J Proteome Res, 14: 185471.http://dx.doi.org/10.1021/pr501221g

Kang, C., Q. Xu, T. D. Martin, M. Z. Li, M. Demaria, L. Aron, T. Lu, B. A. Yankner, J. Campisi, and S. J. Elledge. 2015. 'The DNA damage response induces inflammation and senescence by $\begin{array}{lllll}\text { inhibiting } \quad \text { autophagy } & \text { of }\end{array}$ aaa5612.http://dx.doi.org/10.1126/science.aaa5612

Khosla, S., J. N. Farr, T. Tchkonia, and J. L. Kirkland. 2020. 'The role of cellular senescence in ageing and endocrine disease', Nat Rev Endocrinol, 16: 263-75.http://dx.doi.org/10.1038/s41574-020$\underline{0335-\mathrm{y}}$

Kowald, A., A. Hamann, S. Zintel, S. Ullrich, E. Klipp, and H. D. Osiewacz. 2012. 'A systems biological analysis links ROS metabolism to mitochondrial protein quality control', Mech Ageing Dev, 133: 331-7.http://dx.doi.org/10.1016/j.mad.2012.03.008

Li, B., P. Du, E. E. Smith, S. Wang, Y. Jiao, L. Guo, G. Huo, and F. Liu. 2019. 'In vitro and in vivo evaluation of an exopolysaccharide produced by Lactobacillus helveticus KLDS1.8701 for the 
alleviative effect on oxidative stress', Food Funct, 10: 170717.http://dx.doi.org/10.1039/c8fo01920g

Li, C., Z. Mo, J. Xie, L. Xu, L. Tan, D. Luo, H. Chen, H. Yang, Y. Li, Z. Su, and Z. Su. 2016. 'Chongcao-Shencha Attenuates Liver and Kidney Injury through Attenuating Oxidative Stress and Inflammatory Response in D-Galactose-Treated Mice', Evid Based Complement Alternat Med, 2016: 3878740.http://dx.doi.org/10.1155/2016/3878740

Li, F., G. Huang, F. Tan, R. Yi, X. Zhou, J. Mu, and X. Zhao. 2020. 'Lactobacillus plantarum KSFY06 on d-galactose-induced oxidation and aging in Kunming mice', Food Sci Nutr, 8: 37989.http://dx.doi.org/10.1002/fsn3.1318

Liu, B., Y. Tu, W. He, Y. Liu, W. Wu, Q. Fang, H. Tang, R. Tang, Z. Wan, W. Sun, and Y. Wan. 2018. 'Hyperoside attenuates renal aging and injury induced by D-galactose via inhibiting AMPKULK1 signaling-mediated autophagy', Aging (Albany NY), 10: $4197-$ 212.http://dx.doi.org/10.18632/aging.101723

Losada, D. M., M. E. Jordani, M. C. Jordani, M. A. Piccinato, C. F. Fina, O. Feres, A. B. Chies, P. R. Evora, and E. Silva O. de Castro. 2014. 'Should preconditioning hyperbaric oxygenation protect the liver against ischemia-reperfusion injury? An experimental study in a rat model', Transplant Proc, 46: 56-62.http://dx.doi.org/10.1016/j.transproceed.2013.10.044

Lv, H., C. H. Han, X. J. Sun, and W. W. Liu. 2016. 'Application of hyperbaric oxygen in liver transplantation', Med Gas Res, 6: 212-18.http://dx.doi.org/10.4103/2045-9912.196903

Mamalis, A., D. H. Nguyen, N. Brody, and J. Jagdeo. 2013. 'The active natural anti-oxidant properties of chamomile, milk thistle, and halophilic bacterial components in human skin in vitro', $J$ Drugs Dermatol, 12: 780-4 
Mo, Z. Z., Y. H. Liu, C. L. Li, L. Q. Xu, L. L. Wen, Y. F. Xian, Z. X. Lin, J. Y. Zhan, J. N. Chen, F. F. Xu, and Z. R. Su. 2017. 'Protective Effect of SFE-CO2 of Ligusticum chuanxiong Hort Against d-Galactose-Induced Injury in the Mouse Liver and Kidney', Rejuvenation Res, 20: 231-43.http://dx.doi.org/10.1089/rej.2016.1870

Newgard, C. B., and N. E. Sharpless. 2013. 'Coming of age: molecular drivers of aging and therapeutic opportunities', J Clin Invest, 123: 946-50.http://dx.doi.org/10.1172/JCI68833

Ozden, T. A., H. Uzun, M. Bohloli, A. S. Toklu, M. Paksoy, G. Simsek, H. Durak, H. Issever, and T. Ipek. 2004. 'The effects of hyperbaric oxygen treatment on oxidant and antioxidants levels during liver regeneration in rats', Tohoku $J$ Exp Med, 203: 25365.http://dx.doi.org/10.1620/tjem.203.253

Palmer, D. M., and J. S. Kitchin. 2010. 'Oxidative damage, skin aging, antioxidants and a novel antioxidant rating system', J Drugs Dermatol, 9: 11-5

Pan, H., W. Feng, M. Chen, H. Luan, Y. Hu, X. Zheng, S. Wang, and Y. Mao. 2021. 'Alginate Oligosaccharide Ameliorates D-Galactose-Induced Kidney Aging in Mice through Activation of the Nrf2 Signaling Pathway', Biomed Res Int, 2021: 6623328.http://dx.doi.org/10.1155/2021/6623328

Park, Y. M., X. Sui, J. Liu, H. Zhou, P. F. Kokkinos, C. J. Lavie, J. W. Hardin, and S. N. Blair. 2015. 'The effect of cardiorespiratory fitness on age-related lipids and lipoproteins', J Am Coll Cardiol, 65: 2091-100.http://dx.doi.org/10.1016/j.jacc.2015.03.517

Paulo, I. C., A. L. Silva, C. Piras, B. F. Ramos, F. R. Zanetti, and D. N. Paulo. 2010. '[Splenic autoimplant and hyperbaric oxygen therapy in rats]', Rev Col Bras Cir, 37: 22633.http://dx.doi.org/10.1590/s0100-69912010000300011 
Peng, H. S., M. B. Liao, M. Y. Zhang, Y. Xie, L. Xu, Y. J. Zhang, X. F. Zheng, H. Y. Wang, and Y. F. Chen. 2014. 'Synergistic inhibitory effect of hyperbaric oxygen combined with sorafenib on hepatoma cells', PLoS One, 9: e100814.http://dx.doi.org/10.1371/journal.pone.0100814

Qian, Y., J. Zhang, X. Zhou, R. Yi, J. Mu, X. Long, Y. Pan, X. Zhao, and W. Liu. 2018. 'Lactobacillus plantarum CQPC11 Isolated from Sichuan Pickled Cabbages Antagonizes d-galactose-Induced Oxidation and Aging in Mice', Molecules, 23.http://dx.doi.org/10.3390/molecules23113026

Ramalho, R. J., P. S. de Oliveira, R. C. Cavaglieri, C. Silva, P. R. Medeiros, D. M. Filho, L. F. Poli-deFigueiredo, and I. L. Noronha. 2012. 'Hyperbaric oxygen therapy induces kidney protection in an ischemia/reperfusion model in rats', Transplant Proc, 44: 23336.http://dx.doi.org/10.1016/j.transproceed.2012.07.020

Rufini, A., P. Tucci, I. Celardo, and G. Melino. 2013. 'Senescence and aging: the critical roles of p53', Oncogene, 32: 5129-43.http://dx.doi.org/10.1038/onc.2012.640

Shchors, K., A. I. Persson, F. Rostker, T. Tihan, N. Lyubynska, N. Li, L. B. Swigart, M. S. Berger, D. Hanahan, W. A. Weiss, and G. I. Evan. 2013. 'Using a preclinical mouse model of high-grade astrocytoma to optimize p53 restoration therapy', Proc Natl Acad Sci U S A, 110: E14809.http://dx.doi.org/10.1073/pnas.1219142110

Shen, D., H. Li, R. Zhou, M. J. Liu, H. Yu, and D. F. Wu. 2018. 'Pioglitazone attenuates aging-related disorders in aged apolipoprotein E deficient mice', Exp Gerontol, 102: 10108.http://dx.doi.org/10.1016/j.exger.2017.12.002

Song, P., Q. Zhao, and M. H. Zou. 2020. 'Targeting senescent cells to attenuate cardiovascular disease progression', Ageing Res Rev, 60: 101072.http://dx.doi.org/10.1016/j.arr.2020.101072

Stepien, K., R. P. Ostrowski, and E. Matyja. 2016. 'Hyperbaric oxygen as an adjunctive therapy in 
treatment of malignancies, including brain tumours', Med Oncol, 33: 101.http://dx.doi.org/10.1007/s12032-016-0814-0

Sun, J. H., Y. M. Liu, T. Cao, and W. Q. Ouyang. 2013. '[Effect of kinetin on ovary and uterus in Dgalactose-induced female mouse model of aging]', Sheng Li Xие Bao, 65: 389-94

Teguh, D. N., R. B. Raap, H. Struikmans, and R. A. van Hulst. 2016. 'Hyperbaric oxygen therapy for chronic bowel dysfunction after pelvic radiotherapy', Lancet Oncol, 17: e130e31.http://dx.doi.org/10.1016/S1470-2045(16)00108-X

Terakado, S., M. Ueno, Y. Tamura, N. Toda, M. Yoshinaga, K. Otsuka, A. Numabe, Y. Kawabata, I. Murota, N. Sato, and Y. Uehara. 2012. 'Sodium alginate oligosaccharides attenuate hypertension and associated kidney damage in Dahl salt-sensitive rats fed a high-salt diet', Clin Exp Hypertens, 34: 99-106.http://dx.doi.org/10.3109/10641963.2011.618196

Tian, W., B. Wu, L. Sun, and Y. Zhuang. 2021. 'Protective effect against d-gal-induced aging mice and components of polypeptides and polyphenols in defatted walnut kernel during simulated gastrointestinal digestion', J Food Sci, 86: 2736-52.http://dx.doi.org/10.1111/1750-3841.15744

Tsai, S. J., and M. C. Yin. 2012. 'Anti-oxidative, anti-glycative and anti-apoptotic effects of oleanolic acid in brain of mice treated by D-galactose', Eur $J$ Pharmacol, 689: 818.http://dx.doi.org/10.1016/j.ejphar.2012.05.018

Verma, R., A. Chopra, C. Giardina, V. Sabbisetti, J. A. Smyth, L. E. Hightower, and G. A. Perdrizet. 2015. 'Hyperbaric oxygen therapy (HBOT) suppresses biomarkers of cell stress and kidney injury in diabetic mice', Cell Stress Chaperones, 20: 495505.http://dx.doi.org/10.1007/s12192-015-0574-3

Wang, B. S., L. J. Juang, J. J. Yang, L. Y. Chen, H. M. Tai, and M. H. Huang. 2012. 'Antioxidant and 
Antityrosinase Activity of Flemingia macrophylla and Glycine tomentella Roots', Evid Based Complement Alternat Med, 2012: 431081.http://dx.doi.org/10.1155/2012/431081

Wang, C., Z. Shen, J. Yu, J. Yang, F. Meng, X. Jiang, and C. Zhu. 2020. 'Protective effects of enzyme degradation extract from Porphyra yezoensis against oxidative stress and brain injury in Dgalactose-induced ageing mice', $\quad B r \quad J \quad$ Nutr, $\quad$ 123: 97586.http://dx.doi.org/10.1017/S0007114519003088

Xing, M., Q. Cao, Y. Wang, H. Xiao, J. Zhao, Q. Zhang, A. Ji, and S. Song. 2020. 'Advances in Research on the Bioactivity of Alginate Oligosaccharides', Mar Drugs, 18.http://dx.doi.org/10.3390/md18030144

Yang, Y., Z. Z. Wu, Y. L. Cheng, W. Lin, and C. Qu. 2019. 'Resveratrol protects against oxidative damage of retinal pigment epithelium cells by modulating SOD/MDA activity and activating Bcl-2 expression', Eur Rev Med Pharmacol Sci, 23: 37888.http://dx.doi.org/10.26355/eurrev_201901_16786

Yu, Y., F. Bai, Y. Liu, Y. Yang, Q. Yuan, D. Zou, S. Qu, G. Tian, L. Song, T. Zhang, S. Li, Y. Liu, W. Wang, G. Ren, and D. Li. 2015. 'Fibroblast growth factor (FGF21) protects mouse liver against D-galactose-induced oxidative stress and apoptosis via activating Nrf2 and PI3K/Akt pathways', Mol Cell Biochem, 403: 287-99.http://dx.doi.org/10.1007/s11010-015-2358-6

Yzydorczyk, C., N. Li, E. Rigal, H. Chehade, D. Mosig, J. B. Armengaud, T. Rolle, A. Krishnasamy, E. Orozco, B. Siddeek, C. Juvet, C. Vergely, and U. Simeoni. 2019. 'Calorie Restriction in Adulthood Reduces Hepatic Disorders Induced by Transient Postnatal Overfeeding in Mice', Nutrients, 11.http://dx.doi.org/10.3390/nu11112796

Zhang, X., J. Z. Wu, Z. X. Lin, Q. J. Yuan, Y. C. Li, J. L. Liang, J. Y. Zhan, Y. L. Xie, Z. R. Su, and Y. 
H. Liu. 2019. 'Ameliorative effect of supercritical fluid extract of Chrysanthemum indicum Linnen against D-galactose induced brain and liver injury in senescent mice via suppression of oxidative stress, inflammation and apoptosis', Journal of Ethnopharmacology, 234: 4456.http://dx.doi.org/10.1016/j.jep.2018.12.050

Zhao, J., Y. Han, Z. Wang, R. Zhang, G. Wang, and Y. Mao. 2020. 'Alginate oligosaccharide protects endothelial cells against oxidative stress injury via integrin-alpha/FAK/PI3K signaling', Biotechnol Lett, 42: 2749-58.http://dx.doi.org/10.1007/s10529-020-03010-z

Zhen, Y. Z., Y. J. Lin, K. J. Li, G. L. Zhang, Y. F. Zhao, M. M. Wang, J. B. Wei, J. Wei, and G. Hu. 2016. 'Effects of rhein lysinate on D-galactose-induced aging mice', Exp Ther Med, 11: 30308.http://dx.doi.org/10.3892/etm.2015.2858

Zhuang, Y., Q. Ma, Y. Guo, and L. Sun. 2017. 'Protective effects of rambutan (Nephelium lappaceum) peel phenolics on $\mathrm{H} 2 \mathrm{O} 2$-induced oxidative damages in $\mathrm{HepG} 2$ cells and d-galactose-induced aging mice', Food Chem Toxicol, 108: 554-62.http://dx.doi.org/10.1016/j.fct.2017.01.022 